\title{
Determination of strain relaxation at the free surface of strained-SiGe film using Convergent Beam Electron Diffraction
}

\author{
Sung-Il Baik and Young-Woon Kim ${ }^{\S}$
}

School of Materials Science and Engineering, Seoul National University

Kwanak-ku Shinlim-dong, Seoul, Republic of KOREA

Strained epitaxial film drew much attention for the past decades because of its capability of controlling bandgap nature of materials using strain conservation. $\mathrm{Si}_{1-\mathrm{x}} \mathrm{Ge}_{\mathrm{x}}$ films on $\mathrm{Si}$ substrate is one of the actively being studied with strained lattices. When the strained layer goes through device fabrication process, it inevitably forms additional free surface, side wall normal to the substrate, which will relax the strain maintained from as-grown state. After Treacy[1] reported the surface relaxation phenomena in the composition-modulated structure using weak beam techniques, many studies were reported in the various systems to measure and propose the possible relaxation mechanisms. When the cross-sectional TEM samples were prepared, one can create similar structure to actual device by creating thin section of strained layer attached to the Si substrate. In this study we report a possible mechanism to relax strain in the stain-preserved layer when free surface was created.

5 different compositions of strained $\mathrm{Si}_{1-\mathrm{x}} \mathrm{Ge}_{\mathrm{x}}$ alloy films (with $\mathrm{x}$ ranging from 0.07-0.2) were grown in CVD system. Retention of the strain was confirmed using high-resolution X-ray mapping. Cross-sectional TEM sample was prepared using typical sample preparation technique, followed by finishing ion milling with $1 \mathrm{kV}, 82^{\circ}$ incidence angle. In order to minimize the ionmilling induced warping, rocking ion milling was used to obtain strong support from the thicker region. Figure 1 (a) shows the Large Angle Convergent Beam Electron Diffraction (LACBED) pattern of the cross-section of $\mathrm{Si}_{0.8} \mathrm{Ge}_{0.2} / \mathrm{Si}(001)$, obtained close to [120] zone axis. One can clearly see the bending of HOLZ line, marked with an arrow, across the interface of Si and SiGe ally layer, which means that the film retain strain in the film. Figure 2 shows the CBED pattern from [120] direction of alloy layer, where splitting of $(4 \overline{2} 6)$ and $(8 \overline{4} 4)$ lines are visible while $(8 \overline{4} 0)$ line doe not show any splitting of the HOLZ. Splitted HOLZ maintains same level of crossing angles with each other. It is generally known that the HOLZ lines shift with strain, but the parallel splitting of the HOLZ lines were not fully understood yet. With extensive simulation, it was found that the parallel splitting is only possible when the crystal is rotated along the axis parallel to the substrate, i.e. $[2 \overline{2} 0]$ direction. Possible configuration of the crystal geometry was shown in the Figure 3 in an exaggerated view. The sides with free surface, created by the ion milling, relax the strain and warped the surface compared to the center unrelaxed section. With this configuration, crystal 
rotation can be simulated. To demonstrate the rotation of the crystal, structure shown in Figure 3 were used to simulate the CBED pattern using JEMS[2] program, which result is shown in Figure 4. Splitting was more evident when the CBED patterns were obtained from the top portion of the film, i.e. away from the interface. In summary, the free surface of strained SiGe alloy film, relaxes strain in a way to release the strain resulting in the warped surface with rotation axis parallel to the substrate.

§ Corresponding author : youngwk@snu.ac.kr

[1] M.M.J. Treacy and J.M. Gibson, J. Vac. Sci. Technol. B4, 1658 (1986)

[2] Java version of Electron Microscopy Software, P.A. Stadelmann, Ultramicroscopy 21131 (1987)

[3] This work was supported by the National Program for Tera-level Nanodevices of Ministry of Science of Technology in Korea.

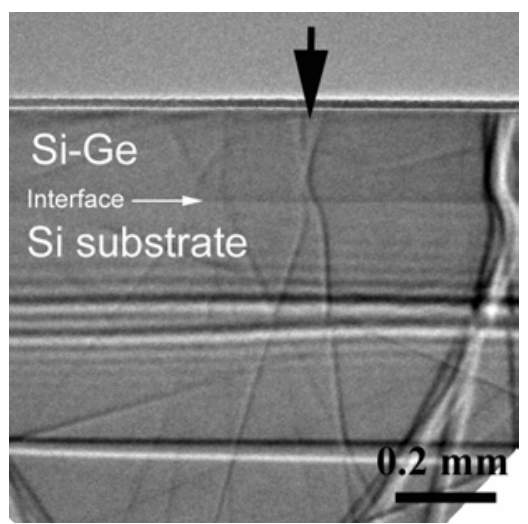

Figure 1. Cross-sectional view of LACBED across the interface

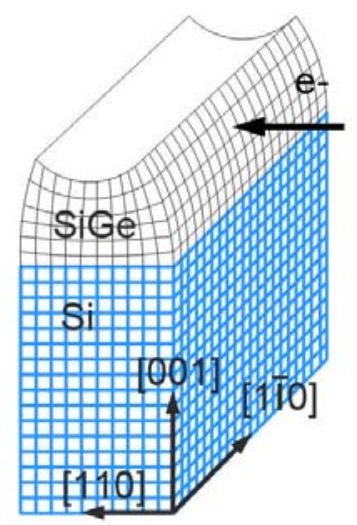

Figure 3. The schematic view of relaxation

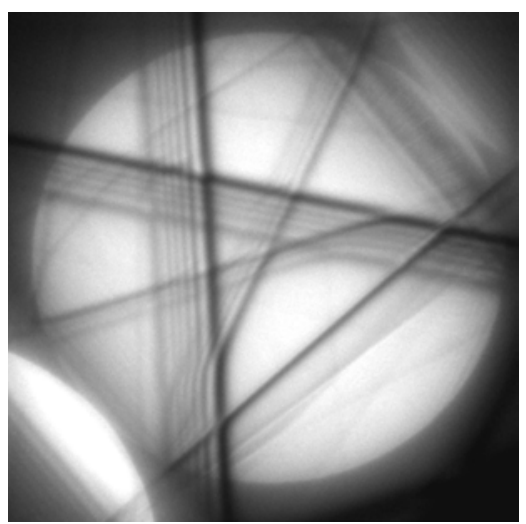

Figure 2. CBED pattern obtained from the top portion of the cross-section

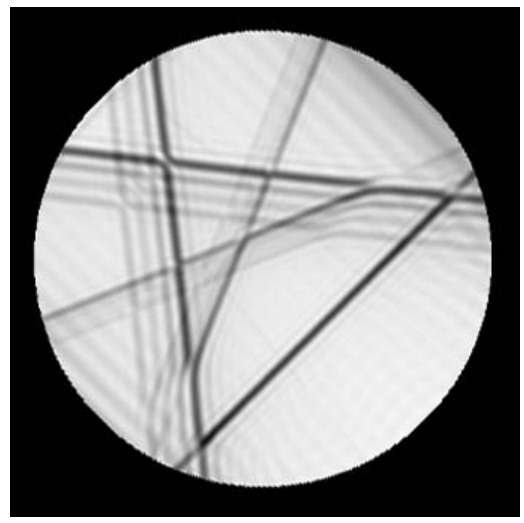

Figure 4. Simulated image of CBED 